\title{
Editors' letter
}

\section{Phyllis Kirstin Illari ${ }^{1} \cdot$ Federica Russo $^{2}$}

Published online: 29 August 2018

(C) Springer Nature B.V. 2018

We have been serving as Editors-in-Chief of EJPS for over a year now, and we thought we would just reflect here on what we have been trying to do in our first year, and alert you to some important upcoming changes.

We have been focusing on three main things in our first year.

Our first and most important aim was to ensure a robust and respectful reviewing process for submissions, and to build an associate editorial team with a shared vision of this. The whole team has put in considerable effort to that, to discussing difficult cases, and helping to build a more collegial and collaborative culture in philosophy of science. We are very happy about how this has gone. This means we are still in favour of discussing submissions thoroughly, rather than deferring objectivity of judgment to a triple-masked system. While there are pros and cons of any system, we feel that, with the Associate Editors, we are providing a fair and solid refereeing process that respects authors' views and values reviewers' work in providing feedback.

Our second important aim was to give visibility to the diversity of sub-fields and approaches within philosophy of science, which is a particularly distinctive feature of European philosophy of science. Our new categorisation for submissions (see also previous editors' letter) seems to be delivering the sought results. Submitted papers are increasingly more diverse in terms of topics and approaches. We don't have hard data on this, but it is noticeable as editors reading every incoming submission. Submissions from women also seem to be growing, although published issues are not yet representative of this. To help maintain cohesion, and help prevent philosophy of science from splintering as a discipline, wherever possible we have also encouraged authors even of highly technical papers to contextualise their work for a broader audience, so that their purposes - if not the technical details - are reasonably widely accessible.

Phyllis Kirstin Illari

phyllis.illari@ucl.ac.uk

Federica Russo

f.russo@uva.nl

1 Department of Philosophy, University of Amsterdam, Amsterdam, Netherlands

2 Department of Science and Technology Studies, University College London, London, UK 
Thirdly, we wished to acknowledge that EJPS is the official journal of EPSA by hosting papers presented at EPSA in Exeter in 2017. This process has been a great success from our point of view. Following an open call for papers, there have been over forty submissions, which have gone through a solid refereeing process. Six papers have now been accepted, and many more are still in the pipeline. More details about the EPSA17 proceedings will be published in a dedicated editorial in due course. We would like to thank authors for submitting, and the guest editors for their hard work in keeping a very high standard of handling submissions.

Keep an eye on EJPS as papers in the Topical Collection of EPSA17 will start appearing in the coming months.

Two big changes are coming in the remainder of 2018.

The first is that EJPS will switch to continuous publication. From late in 2018, EJPS won't have the old issues with a limited number of papers. Instead, papers will be published online on a continuous basis, so authors do not need to wait to cite the article as published. Paper issues will still be collected three times a year, but simply contain all papers fully accepted since the previous issue. This speeds up publication, so that there will be no backlog. It also offers us more flexibility in terms of how we can use online resources for managing the journal.

An example of this flexibility lies in the second big change. EJPS will start offering 'Topical Collections'. These are not unlike special issues, which are widely prepared and published in our field, although at times their quality is contested. EJPS published a few in the past, but they were discontinued.

We have created a clear editorial policy on Topical Collections, which we hope will create what is valuable in special issues. Topical collections can shape or re-shape a debate, or give visibility to a new topic or approach, and these are all academically valuable. Yet this value must come with high quality, and with an open process that resists simply replicating existing work and prestige. We will issue an open call for proposals for Topical Collections late in 2018. Submissions will be assessed by the Editors-in-Chief and Associate Editors as appropriate given their expertise. Papers in Topical Collections will undergo the same refereeing process as any other EJPS submission, with Guest Editors acting as Associate Editors and handling all papers via the Editorial Manager. The Editorsin-Chief must confirm all final decisions, and will have oversight on the whole process. This is a procedure that has worked extremely well for the EPSA17 track articles, which will form the first Topical Collection of EJPS. We will start accepting Topical Collection proposals in late 2018 or early 2019 . Keep an eye on the website, email lists, and twitter for further information and links to more detailed guidance for proposals.

We would like to finish by thanking the many people who have worked with us on EJPS. Most immediately, these are the Associate Editors and the Guest Editors of the EPSA17 track papers, who have somehow managed to be both reliable and inspiring. We also wish to thank Ties Nijssen of Springer for a lot of work and support, particularly on both the switch to continuous publication, and the forming of policies for Topical Collections. Thanks also to Lucy Fleet and Jennyca Parcon also of Springer for a good deal of work. Finally, thank you to the many reviewers, who do the most astonishingly important and collegial work, even while hidden behind the scenes.

Phyllis Illari and Federica Russo

Editors in Chief 